\title{
Barrow holographic dark energy with Hubble horizon as IR cutoff
}

\author{
Shikha Srivastava ${ }^{1}$, Umesh Kumar Sharma $^{2}$ \\ 1,2 Department of Mathematics, Institute of Applied Sciences and Humanities, GLA University \\ Mathura-281406, Uttar Pradesh, India \\ ${ }^{1}$ E-mail: shikha.azm06@gmail.com \\ ${ }^{2}$ E-mail: sharma.umesh@gla.ac.in
}

\begin{abstract}
In this work, we propose a non-interacting model of Barrow holographic dark energy (BHDE) using Barrow entropy in a spatially flat FLRW Universe considering the IR cutoff as the Hubble horizon. We study the evolutionary history of important cosmological parameters, in particular, EoS $\left(\omega_{B}\right)$, deceleration parameter and, the BHDE and matter density parameter and also observe satisfactory behaviours in the BHDE the model. In addition, to describe the accelerated expansion of the Universe the correspondence of the BHDE model with the quintessence scalar field has been reconstructed.
\end{abstract}

Keywords : BHDE, FLRW, quintessence

PACS: 98.80.-k

\section{Introduction}

According to a sequence of past and latest observational data [1-6], the dark sector of our Universe is filled with two dark fluids, namely the dark energy (DE) and dark matter (DM). The former fluid drives the current accelerating stage of the Universe while the later is responsible for the structure formation of the Universe. It is also observed from the observational data that about ninety-six per cent of the total energy density of the Universe is coming from this combined dark component, where the contribution of DE is around sixty-eight per cent of the total energy allocation of the Universe while the DM contributes approximately twenty-eight per cent of the total energy allocation of the Universe. Although, the origin, evolution and the characteristics of these dark sector of the DE are not distinctly recognized yet [7-12]. However, the nature of DM appears to be partially known by indirect gravitational effects, although, the DE has endured being exceptionally mysterious. As a consequence, in the last couple of years, various cosmological models have been proposed and explored. The non-interacting models of the Universe are considered to be the simplest model leading to two independent evolution of these dark components where DE and DM are conserved separately. In a more general form of available cosmological models interaction between DE and DM are permitted. [13].

The quantitative description of dark energy can suitably be substituted by holographic dark energy (HDE) [14, 15], deriving from holographic principle (HP) [16 20]. Notably one can 
recieve the consequence of vacume energy of holographic source at astrophysical scales structure DE [14,15] due to the relationship between the longest length of QFT (quantum field theory) with its UV cutoff [21]. Interestly both the simple [22 31] and extended [32 55] versions of HDE represents to lead to a fascinating cosmological behaviour and they also constrained with observations 56 64]. The Universe horizon entropy remains proportional to its area, which becomes the most pevetel step in the application of HP at cosmological context, similar to a black hole BH (Bekenstein-Hawking) entropy. Although, for the black-hole structure, recently Barrow propoesd that the QG (quantum-gravitational) impacts can lead to introduce fractal, intricate behaviours. He emphasized that this complex structure gives finite volume but with the finite (or infinite) area and hence a deformed expression of black-hole entropy [65]

$$
S_{B}=\left(\frac{A}{A_{0}}\right)^{\frac{2+\Delta}{2}},
$$

where, $A_{0}$ and $A$ are Planck area and standard horizon area, respectively. A new exponent $\Delta$, contributing to the quantum-gravitational deformation was in $\Delta=1$ corresponding to its the most fractal and intricate structure and corresponds to the usual BH entropy when $\Delta=$ 0 . It is important to mention that the standard "quantum-corrected" entropy with corrections logarithmic 66, 67] is different from the above quantum-gravitationally corrected entropy. However, it resembles non-extensive Tsallis entropy [68 70], but the physical principles and involved foundations are totally different.

Recently, Saridakis [71], constructed the BHDE, by using the usual HP, however applying the Barrow entropy instead of the $\mathrm{BH}$ entropy. Also, for the limiting case as $\Delta=0$, the BHDE possesses standard HDE, although The BHDE, in general, is a new scenario with nice cosmological behavior and richer structure. While standard HDE is given by the inequality $\rho_{B} L^{4} \leq S$, where $L$ denotes horizon length, and under the imposition $S \propto A \propto L^{2}[14]$, the Eq. (1) will give

$$
\rho_{B}=C L^{\Delta-2}
$$

where $C$ is a parameter having $[L]^{-2-\Delta}$ dimensions $[71$. As expected, in the case $\Delta=0$, the above expression gives the standard HDE $\rho_{B}=3 c^{2} M_{p}^{2} L^{-2}$, where $C=3 c^{2} M_{p}^{2}$ with $c^{2}$ the model parameter and $M_{p}$ is the Planck mass. Depending on the parameter $\Delta$, the BHDE will deviate by the standard one, which may lead to distinct cosmological behaviour. If we take into consideration the IR cut off $L$ as the Hubble horizon $\left(H^{-1}\right)$, then the energy density of BHDE is obtained as

$$
\rho_{B}=C H^{2-\Delta} \text {. }
$$

Saridakis [72], using Barrow entropy presented a modified cosmological scenario besides the Bekenstein-Hawking one. For the evolution of the effective DE density parameter, the analytical expression was obtained and shown the DM to DE era of the Universe. Using the Barrow entropy on the horizon in place of the standard Bekenstein-Hawking one, the potency of the generalized second law of thermodynamics has also been examined [73. Mamon et al. 74 studied interacting BHDE model and also the validity of the generalized second law by assuming dynamical apparent horizon as the thermodynamic boundary. More recently, Anagnostopoulos et al. 75 have shown that the BHDE is in concurrence with observational information, and it can serve as a decent contender for the depiction of DE. The authors examined the Tsallis 
nonextensive form of the logarithmically amended Barrow entropy $[76]$. Barrow's new idea of entropy have some significant examinations $[77,78]$. Now, we discuss the similarity and difference with other work in literature. Recently, an interacting model of the BHDE has been proposed by using Barrow entropy. In particular, the evolution of a spatially flat FLRW Universe filled with BHDE and pressureless DM that interact with each other through a well-motivated interaction term has been investigated by taking the Hubble horizon as IR cutoff [74]. While in this work we focus the BHDE model without interaction in a flat FLRW Universe with Hubble horizon as IR cutoff. We organize the present work in the following way. The cosmological parameters of BHDE model are discussed in Sect. 2. In Sect. 3, we investigate the stability of the BHDE model. The correspondence between the BHDE and quintessence scalar field model and also potentials for scalar field models are discussed in Sect. 4. In Sect. 5, we draw our conclusions.

\section{The Cosmological Model}

The first Friedmann equation for a flat FRW Universe which is composed by pressureless DM $\left(\rho_{m}\right)$ and $\operatorname{BHDE}\left(\rho_{B}\right)$ is

$$
H^{2}=\frac{1}{3 m_{p}^{2}}\left(\rho_{B}+\rho_{m}\right),
$$

expounding the dimensionless density parameter as $\Omega_{i}=\rho_{i} / \rho_{c}$, where $\rho_{c}=3 m_{p}^{2} H^{2}$ is known as the critical energy density, we can obtain

$$
\Omega_{B}=\frac{\rho_{B}}{3 m_{p}^{2} H^{2}}=\frac{C}{3 m_{p}^{2}} H^{-\Delta}, \quad \Omega_{m}=\frac{\rho_{m}}{3 m_{p}^{2} H^{2}} .
$$

The conservation law corresponding to dust and BHDE are

$$
\begin{gathered}
\dot{\rho}_{m}+3 H \rho_{m}=0, \\
\dot{\rho}_{B}+3 H \rho_{B}\left(1+\omega_{B}\right)=0 .
\end{gathered}
$$

Here $\omega_{B}=p_{B} / \rho_{B}$ is EoS parameter and $p_{B}$ is pressure of BHDE. Differentiating Eq. (4) with respect to time and solving Eqs. (6), (7) and Eq. (5), we can obtain

$$
\frac{\dot{H}}{H^{2}}=-\frac{3}{2}\left(1+\omega_{B} \Omega_{B}\right) .
$$

Substituting Eq. (3) in Eq. (7), we will find

$$
\frac{\dot{H}}{H^{2}}=-\frac{3\left(1+\omega_{B}\right)}{2-\Delta},
$$

by the help of Eq. (7) and Eq. (8), we get

$$
\omega_{B}=-\frac{\Delta}{2-(2-\Delta) \Omega_{B}} .
$$

From Eq. (8), we can find that

$$
\Omega_{B}^{\prime}=\frac{d \Omega_{B}}{d(\ln a)}=(1-\Delta) \Omega_{B} \frac{\dot{H}}{H^{2}} .
$$




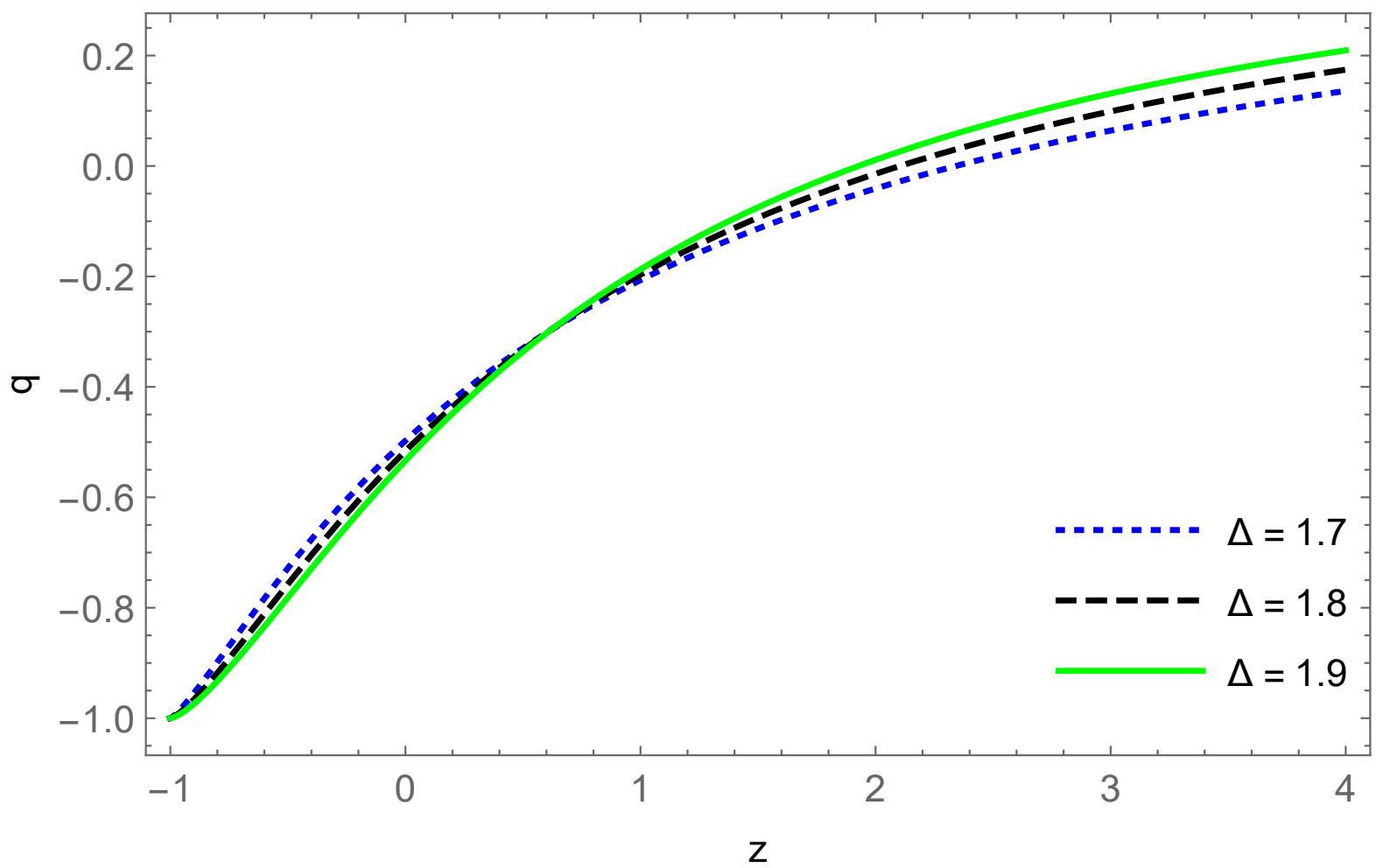

Figure 1: Expansion of deceleration parameter verses redshift $z$ distinct estimations of $\Delta$. Here, we take $\Omega_{B 0}=0.70$.

Associating the above result with Eqs. (9) and (10), we obtains

$$
\Omega_{B}^{\prime}=-3(1-\Delta)\left(\frac{1-\Omega_{B}}{2-(2-\Delta) \Omega_{B}}\right) \Omega_{B}
$$

As we know that deceleration parameter is given as

$$
q=-\left[1+\frac{\dot{H}}{H^{2}}\right]
$$

putting Eq. (9) in Eq. (13) and Using Eq. (10), we get

$$
q=\left[\frac{1-(1+\Delta) \Omega_{B}}{2-(2-\Delta) \Omega_{B}}\right] .
$$

The evolutionary behaviour of the deceleration parameter is plotted for the BHDE model versus redshift $z$ by finding its numerical solution using the initial values of $\Omega_{B}$ as $\Omega_{B 0}=0.70$. The value of deceleration parameter $q$ decides the nature of the Universe such as if $q<0$, it is accelerating and if $q>0$, it is in decelerating phase. It is proposed by various observations that the Universe is in an accelerated expansion phase and the value of deceleration parameter lies in the range $-1 \leq q<0$. The evolutionary behaviour of the deceleration parameter versus redsfit $z$ is plotted in Fig. 1. We observe from this figure that the deceleration parameter of the BHDE model transits from an early decelerated phase to the current accelerated phase for the different values of the parameter $\Delta$. The evolutionary behaviour of the EoS parameter $\omega_{B}$ 


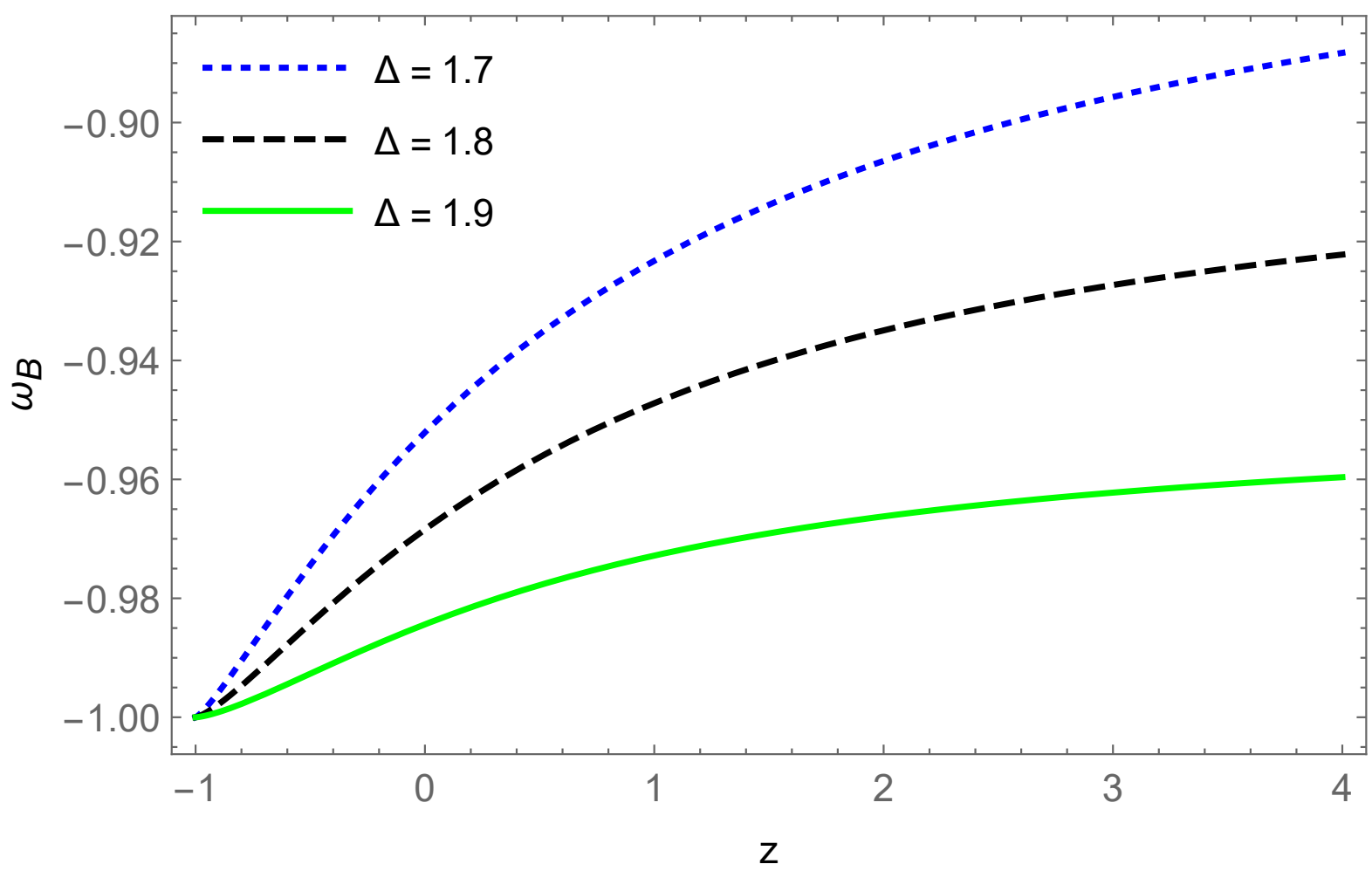

Figure 2: The behaviour of the EoS parameter of BHDE $\omega_{B}$ against $z$ for different $\Delta$. Here, we take $\Omega_{B 0}=0.70$.

versus $z$ for the BHDE model is plotted in Fig. 2 for the different values of the parameter $\Delta$. We can observe that the EoS parameter of the BHDE remains in quintessence era, and approaches to the cosmological constant $\left(\omega_{B}=-1\right)$ at future for $\Delta=1.7,1.8$ and 1.9. It is significant that EoS parameter of the BHDE gives pleasant conduct and it tends to be quintessence-like for the various estimations of $\Delta$.

The modified cosmological scenario has been constructed by applying the FLT (first law of thermodynamics) and using the Barrow entropy [72]. Moreover, Saridakis [72], proposed this cosmological modified scenario with varying parameter $\Delta$ by applying the non-extensive thermodynamics. In [71 73], the researcers proposed that this cosmological modified scenario gives a description of both inflation and late-time acceleration of the Universe with varying parameter from the FLT. In Fig. 3(a) and 3(b) we have plotted the energy density for the BHDE $\Omega_{B}$ and energy density of matter $\Omega_{m}$ as a function of redshift. The thermal history of the Universe, in particular, the successive sequence of matter and DE era can be observed from these figures. In the papers [71-73], it has also been proposed by some of the researchers, relating to the late times Universe evolution, a new term that appear due to the varying non-extensive exponent constituted an effective DE sector. Futher, it has been shown that Universe shows usual thermal history, with the successive sequence of matter and De epochs, and the transition to acceleration is in agreement with the observed behavior. 


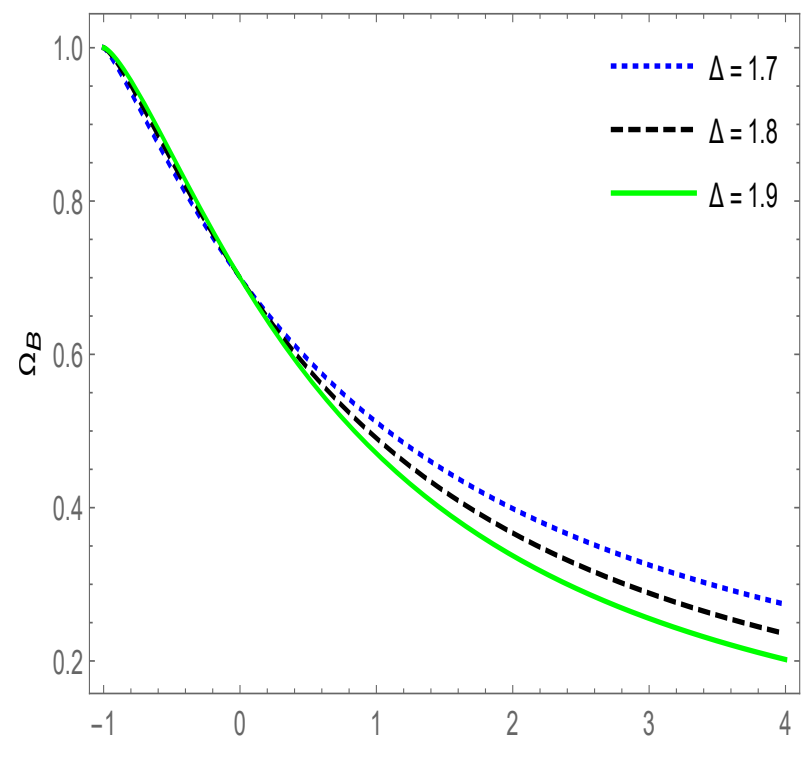

(a)

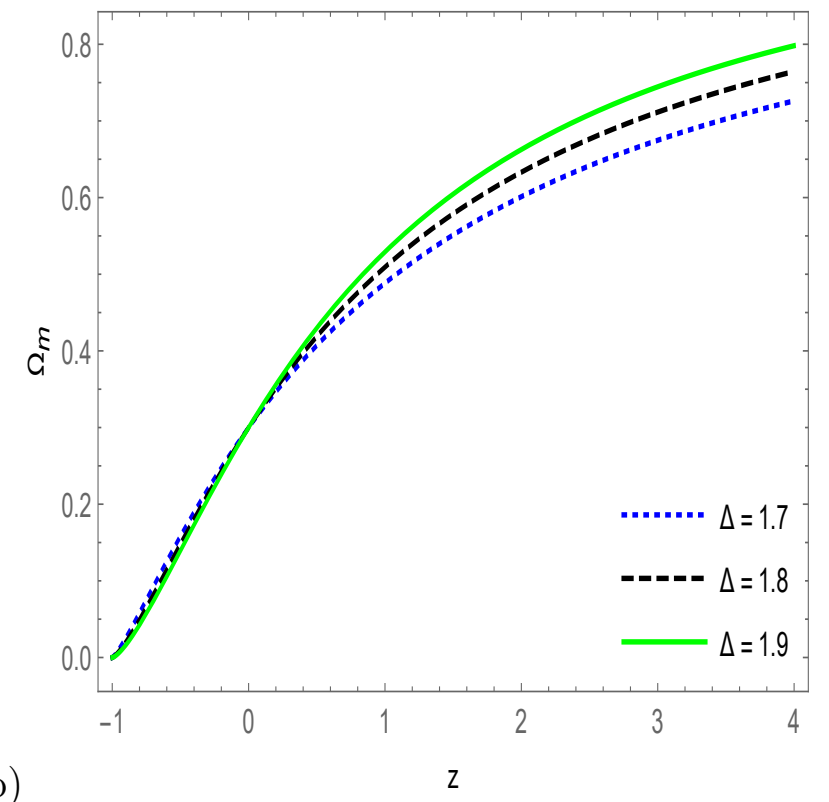

(b)

Figure 3: (a) Expansion of BHDE density parameter $\Omega_{B}$ with $z$ redshift for distinct value of $\Delta$.Here, we take $\Omega_{D 0}=0.70$ (b) The evolution of matter energy density parameter $\Omega_{m}$ with $z$ (redshift) for distinct value of $\Delta$. Here, we take $\Omega_{B 0}=0.70$.

\section{Analysis of BHDE Model for its Stability}

Squared sound speed lies in a range $0 \leq v_{s}^{2} \leq 1$ physically [79. We take EoS parameter $\omega_{B} \neq-1$ to analyse the impact of the DE sound speed, because dark energy is of the form of cosmological constant consist of no perturbations so there is lack of sensitivity to the DE speed of sound squared. Effect of sound speed of dark energy on the CMB power spectrum is detailed has analysed in [80] concluding that there is no impact of LISW (late-time integrated Sachs-Wolfe) with the decrement of $v_{s}^{2}$ from 1 to 0 and when $\omega_{B}>-1$ but when $\omega_{B}<-1$ LISW gives positive impact. Consequently, we can see more clustering of (cold) dark energy with a decrement in $\omega_{B}>-1$ resulting in an increment of dark energy perturbations which is having the power to stop the potential to vanish and preserve it and thus introducing the LISW effect a small push 79$]$.

Stability Test for BHDE Model against the perturbation has been done using squared speed of sound $v_{s}^{2}=\frac{d p_{T}}{d \rho_{B}}, 81$. Generally, we give the squared speed of sound in the form:

$$
v_{s}^{2}=\frac{d p_{B}}{d \rho_{B}}=\frac{2 \Delta(1-\Delta) \Omega_{B}-2 \Delta+\Delta \Omega_{B}}{\left[2-(2-\Delta) \Omega_{B}\right]^{2}} .
$$




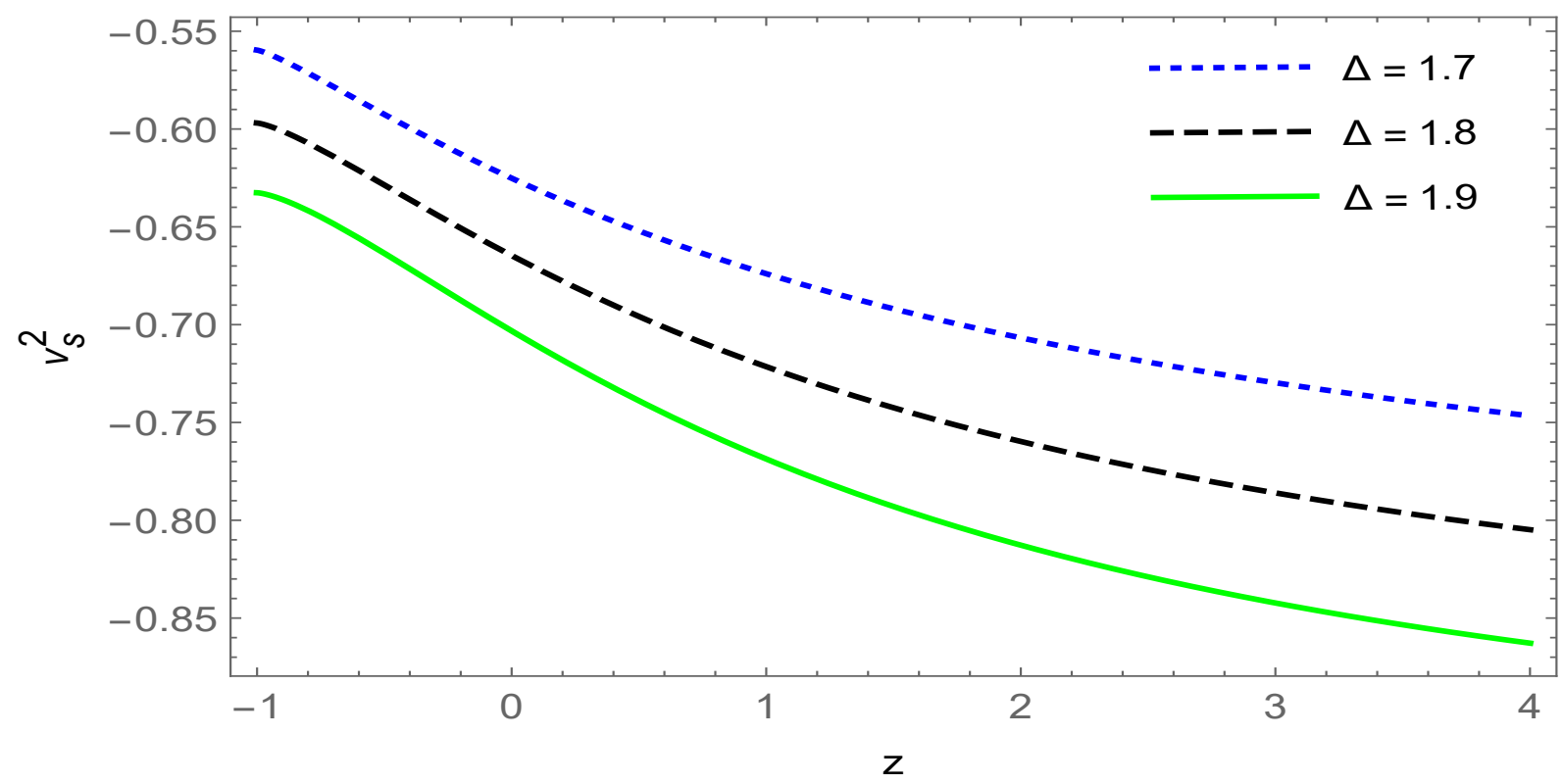

Figure 4: The behaviour of squared sound speed $v_{s}^{2}$ verses redshift $z$ for distinct value of $\Delta$. Here, we take $\Omega_{B 0}=0.70$.

The squared speed of sound $v_{s}^{2}$ represented by Eq. (15), is plotted in Fig. 4 as a function of redshift $(z)$ for different values of the parameter $\Delta$, and it can be observed by the figure that for $\Delta<2$, The BHDE model is unstable during the cosmic evolution.

\section{The correspondence of BHDE with quintessence field}

In view of this segment, we will deliberate the correspondence of the BHDE with quintessence scalar field model. The potentials and dynamics for quintessence scalar field are also reconstructed. By comparing energy densities of the scalar field models and the BHDE model given in Eq. (3), we obtain the correspondence. Here we also compare the EoS parameter for scalar fields (quintessence ) with EoS of BHDE model stated by Eq. (10). Both the dark energy, canonical and non-canonical are well described by using scalar fields. In the present article, we have chosen the quintessence field as canonical. As we know that for quintessence scalar field $\omega_{B}>-1[82[86]$.

The pressure and energy density for quintessence scalar field [87] are respectively given below as:

$$
p=\frac{\dot{\phi}^{2}}{2}-V(\phi), \rho=\frac{\dot{\phi}^{2}}{2}+V(\phi),
$$

where $V(\phi)$ stands for scalar field potential and $\phi$ stands for scalar field.

$$
\begin{aligned}
& V(\phi)=\frac{1-\omega_{B}}{2} \rho_{B}, \\
& \dot{\phi}^{2}=\left(1+\omega_{B}\right) \rho_{B} .
\end{aligned}
$$




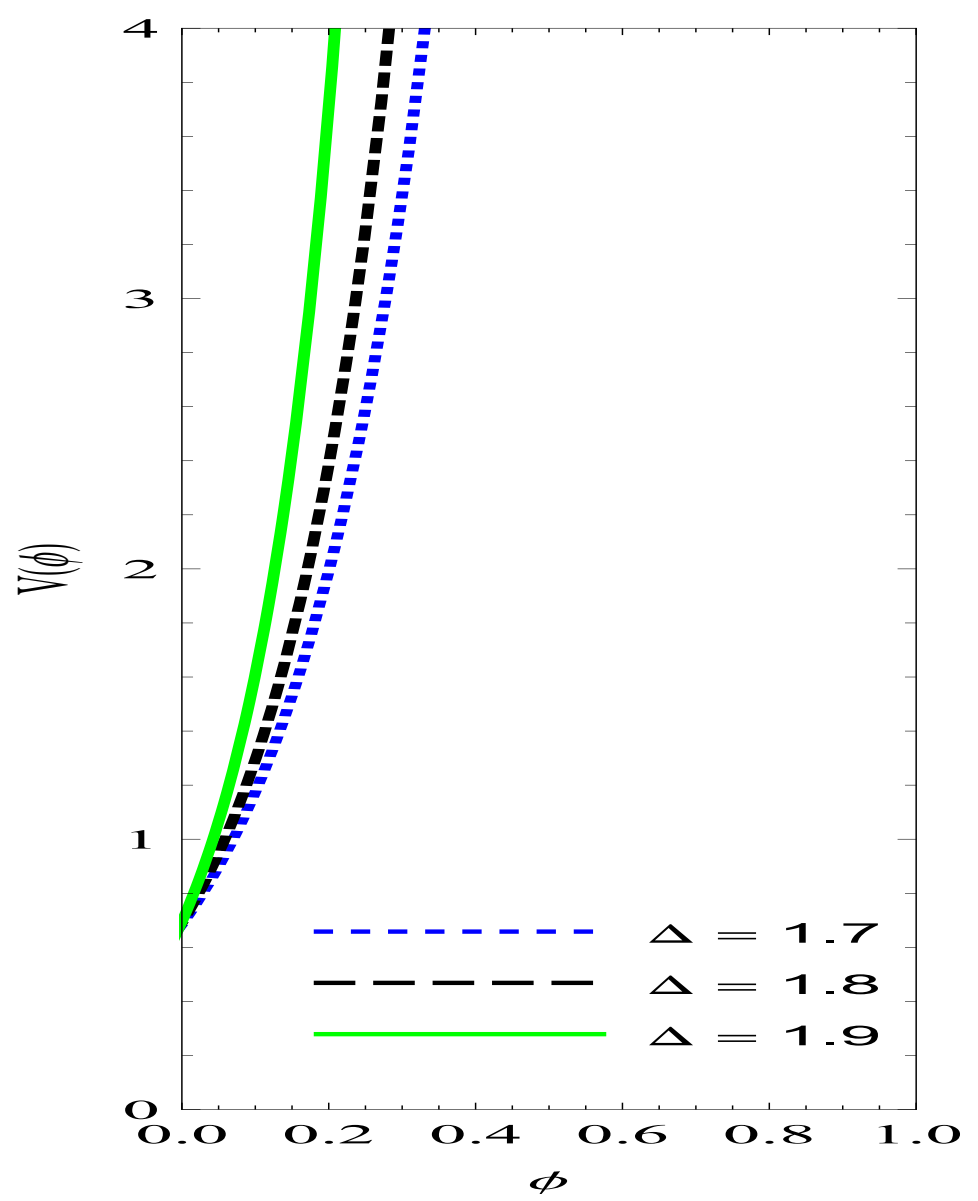

Figure 5: The potential reconstruction for the Barrow holograhpic quintessence for different $\Delta$, here $V(\phi)$ and $\phi$ are taken in unit of $\rho_{c 0}$ and $M_{p}^{2}$ respectively and $\Omega_{B 0}=0.70$.

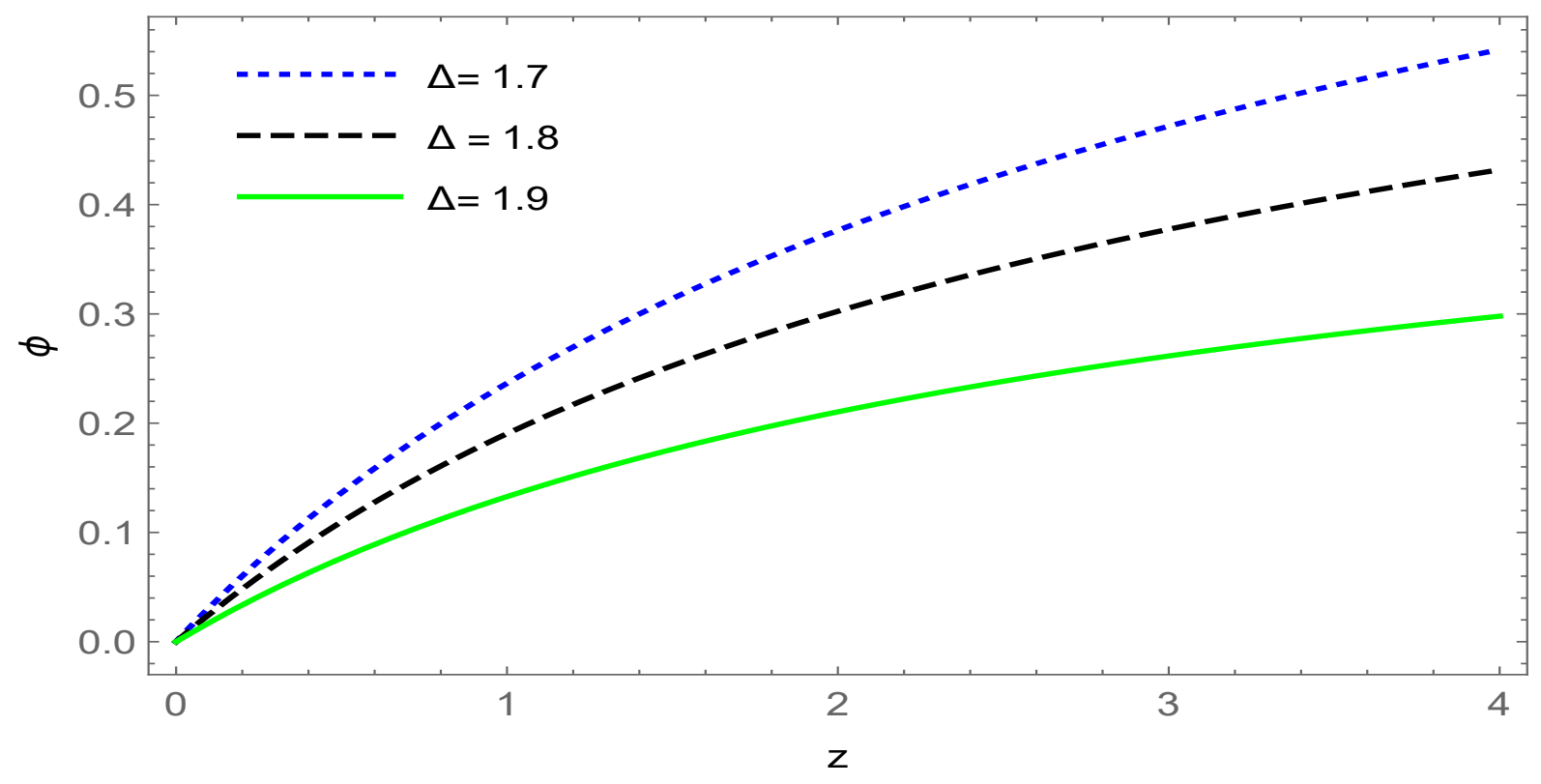

Figure 6: The evolution of the scalar-field $\phi(z)$ for quintessence of Barrow holograhpic, here $\phi$ is taken in unit of $M_{p}^{2}$ and $\Omega_{B 0}=0.70$. 
Moreover, from the equation of a flat FLRW $3 M_{p}^{2} H^{2}=\rho_{m}+\rho_{B}$, we can obtain

$$
E(z) \equiv \frac{H(z)}{H_{0}}=\left(\frac{\Omega_{m 0}(1+z)^{3}}{\left(1-\Omega_{B}\right)}\right)^{1 / 2},
$$

here $\Omega_{m 0}$ represents the present fractional energy density of pressureless matter. By the help of Eq. (19) we can express Eqs. (17) and (18) respectively 87

$$
\begin{aligned}
\frac{V(\phi)}{\rho_{c 0}} & =\frac{1}{2}\left(1-\omega_{B}\right) \Omega_{B} E^{2}, \\
\frac{\dot{\phi}^{2}}{\rho_{c 0}} & =\left(1+\omega_{B}\right) \Omega_{B} E^{2} .
\end{aligned}
$$

Where $\rho_{c 0}=3 M_{P}^{2} H_{0}^{2}$ gives present critical density of the Universe. For the propose correspondence between the BHDE and the quintessence scalar field, namely, in particular, we recognize $\rho$ with $p$. Futhermore, quintessence field takes over Barrow nature such as $E, \Omega_{B}$ and $\omega_{B}$ are represented by Eqs. (10), (12) and (19). According to [87], we assume $\dot{\phi}>0$ in this paper. Differentiating $\phi$ (scalar field) with respect to $z$ (redshift), given as

$$
\frac{\frac{d \phi}{d z}}{M_{p}}=\frac{\sqrt{3\left(1+\omega_{B}\right) \Omega_{B}}}{1+z} .
$$

By integrating Eq. (22), we can without much of a stretch acquire the evolutionary structure of the field as :

$$
\phi(z)=\int_{0}^{z} \frac{d \phi}{d z} d z,
$$

here, the field amplitude $(\phi(z))$, at the present epoch $(z=0)$ is constant to be zero such as $\phi(0)=0$. In this manner, this can be constituted as Barrow Holographic quintessence DE model and recreate the potential of the BHDE.

In Fig. $5, V(\phi)$, the reconstructed quintessence potential is graphed, and $\phi(z)$ which is also recreated by Eqs. (22) and (23), plotted in Fig. 6. Assuming present fractional energy density is $\Omega_{\mathrm{B} 0}=0.70$., chosen curves are portrayed for the different cases of $\Delta$. The dynamics of the scalar field explicitly observed from Figs. 5 and 6. Clearly, the scalar field $\phi$ moves down the potential with kinetic energy $\dot{\phi}^{2}$ slowly diminishing.

\section{Conclusions}

In this paper, we have constructed the BHDE model without interaction which depends on Barrow entropy proposed by Barrow recently, it begins with the modification proposal of the black-hole structure because of some QG effects. We have studied the evolution of a spatially flat FLRW Universe composed of the BHDE and pressureless DM by considering the IR cutoff as Hubble horizon. We have explored the behaviour of cosmological parameters such as DP (deceleration parameter), the EoS parameter, the BHDE and matter-energy density parameter during the cosmic evolution. The correspondence between BHDE model and the quintessence 
scalar field models have also been done to clarify the late-time cosmic accelerated expansion of the Universe. We finish up our outcomes as follows :

- It has been observed that the BHDE model exhibits a smooth transformation from an early deceleration era to present acceleration era of the Universe and in a good agreement with recent cosmic observations.

- The behaviour of EoS parameter can be observed by varying the exponent $\Delta$. The EoS parameter of the BHDE model varies in the quintessence region for different values of the parameter $\Delta$.

- We extricated a straightforward DE (Differential Equation) for the evolution of the DE density parameter, and we presented the behaviour of BHDE and matter density parameter. As we appeared, the situation of the BHDE can portray the Cosmos usual history, with the arrangement of matter and DE era.

- We observe that for $\Delta<2$, the BHDE model is not stable for all values of $z$ during the cosmic evolution. It can be fixed by taking other IR cutoffs, probable interactions between the Universe sectors, different entropy corrections or even a mix of these scenarios. Indeed, this contemplation can likewise modify and increase predictions and behaviour of BHDE. These are subjects concentrated in future to turn out to be all the more near the various properties of BHDE, and thus the cause of the DE.

- We proposed a correspondence between BHDE model and quintessence scalar-field model. We have a look at that the BHDE with $\Delta<2$ may be described absolutely with the aid of the quintessence in a sure manner. The correspondence between BHDE and quintessence has been hooked up, and the potential of the Barrow holographic quintessence and the dynamics of the field have been recreated.

\section{Acknowledgements}

The author S. Srivastava thankfully recognize the utilization of administrations and office gave by GLA University, Mathura, India to lead this research work. The author U. K. Sharma thanks the IUCAA, Pune, India for awarding the visiting associateship.

\section{References}

[1] S. Perlmutter et al. [Supernova Cosmology Project Collaboration], "Measurements of $\Omega$ and $\Lambda$ from 42 high redshift supernovae," Astrophys. J. 517 (1999) 565-586.

[2] A. G. Riess et al. [Supernova Search Team], "Observational evidence from supernovae for an accelerating universe and a cosmological constant," Astron. J. 116 (1998), 1009-1038.

[3] P. Ade et al. [Planck], "Planck 2015 results. XIII. Cosmological parameters," Astron. Astrophys. 594 (2016), A13. 
[4] T. Abbott et al. [DES], "First Cosmology Results using Type Ia Supernovae from the Dark Energy Survey: Constraints on Cosmological Parameters," Astrophys. J. Lett. 872 (2019) no.2, L30.

[5] N. Aghanim et al. [Planck Collaboration], "Planck 2018 results. VI. Cosmological parameters," arXiv:1807.06209 [astro-ph.CO].

[6] L. Amendola et al., "Cosmology and fundamental physics with the Euclid satellite," Living Rev. Rel. 21 (2018) no.1, 2.

[7] S. Capozziello, "Dark Energy Models toward observational tests and data," Int. J. Geom. Meth. Mod. Phys. 4(01) (2007), 53.

[8] S. Capozziello, V. Cardone and A. Troisi, "Dark energy and dark matter as curvature effects," JCAP 08 (2006) 001.

[9] C. Escamilla-Rivera, M. A. C. Quintero and S. Capozziello, "A deep learning approach to cosmological dark energy models," JCAP 03 (2020) 008.

[10] S. Capozziello, Ruchika and A. A. Sen, "Model independent constraints on dark energy evolution from low-redshift observations," Mon. Not. Roy. Astron. Soc. 484 (2019), 4484

[11] S. Capozziello, V. F. Cardone, A. Troisi, " Reconciling dark energy models with $f(R)$ theories," Phys. Rev. D 71(4), (2005) 043503.

[12] S. Capozziello, V. F. Cardone, E. Elizalde, S. Nojiri and S. D. Odintsov, "Observational constraints on dark energy with generalized equations of state," Phys. Rev. D 73 (2006), 043512 .

[13] L. Amendola, "Coupled quintessence," Phys. Rev. D 62 (2000), 043511.

[14] M. Li, "A Model of holographic dark energy," Phys. Lett. B 603 (2004), 1.

[15] S. Wang, Y. Wang and M. Li, "Holographic Dark Energy," Phys. Rept. 696 (2017), 1-57.

[16] L. Susskind, "The World as a hologram," J. Math. Phys. 36 (1995), 6377-6396. arXiv:hepth/9409089 [hep-th]]

[17] W. Fischler and L. Susskind, "Holography and cosmology," arXiv:hep-th/9806039 [hepth]].

[18] G. 't Hooft, "Dimensional reduction in quantum gravity," Conf. Proc. C 930308 (1993), 284-296 arXiv:gr-qc/9310026 [gr-qc]]..

[19] P. Horava and D. Minic, "Probable values of the cosmological constant in a holographic theory," Phys. Rev. Lett. 85 (2000), 1610-1613 [hep-th/0001145].

[20] R. Bousso, "The Holographic principle," Rev. Mod. Phys. 74 (2002), 825-874.

[21] A. G. Cohen, D. B. Kaplan and A. E. Nelson, "Effective field theory, black holes, and the cosmological constant," Phys. Rev. Lett. 82 (1999), 4971-4974. 
[22] D. Pavon and W. Zimdahl, "Holographic dark energy and cosmic coincidence," Phys. Lett. B 628 (2005), 206-210.

[23] B. Wang, C. Y. Lin and E. Abdalla, "Constraints on the interacting holographic dark energy model," Phys. Lett. B 637 (2006), 357-361.

[24] R. Horvat, "Holography and variable cosmological constant," Phys. Rev. D 70 (2004), 087301.

[25] M. R. Setare and E. N. Saridakis, "Correspondence between Holographic and GaussBonnet dark energy models," Phys. Lett. B 670 (2008), 1-4.

[26] Q. G. Huang and M. Li, "The Holographic dark energy in a non-flat universe," JCAP 08 (2004), 013.

[27] S. Nojiri and S. D. Odintsov, "Unifying phantom inflation with late-time acceleration: Scalar phantom-non-phantom transition model and generalized holographic dark energy," Gen. Rel. Grav. 38 (2006), 1285-1304.

[28] B. Wang, Y. g. Gong and E. Abdalla, "Transition of the dark energy equation of state in an interacting holographic dark energy model," Phys. Lett. B 624 (2005), 141-146.

[29] M. R. Setare, "Interacting holographic dark energy model in non-flat universe," Phys. Lett. B 642 (2006), 1-4.

[30] M. R. Setare and E. N. Saridakis, "Non-minimally coupled canonical, phantom and quintom models of holographic dark energy," Phys. Lett. B 671 (2009), 331-338.

[31] H. Kim, H. W. Lee and Y. S. Myung, "Equation of state for an interacting holographic dark energy model," Phys. Lett. B 632 (2006), 605-609.

[32] E. N. Saridakis, "Holographic Dark Energy in Braneworld Models with a Gauss-Bonnet Term in the Bulk. Interacting Behavior and the $\mathrm{w}=-1$ Crossing," Phys. Lett. B 661 (2008), 335-341.

[33] M. Tavayef, A. Sheykhi, K. Bamba and H. Moradpour, "Tsallis Holographic Dark Energy," Phys. Lett. B 781 (2018), 195.

[34] L. P. Chimento and M. G. Richarte, "Dark radiation and dark matter coupled to holographic Ricci dark energy," Eur. Phys. J. C 73 (2013) no.4, 2352.

[35] A. Jawad, S. Husain, S. Rani and S. Qummer, "Generalized ghost Tsallis holographic dark energy model in RS-II braneworld and dynamical Chern-Simons modified gravity," Int. J. Geom. Methods Mod. Phys. (2020), doi: 10.1142/S0219887820501248.

[36] H. Moradpour, S. A. Moosavi, I. P. Lobo, J. P. Morais Graça, A. Jawad and I. G. Salako, "Thermodynamic approach to holographic dark energy and the Rényi entropy," Eur. Phys. J. C 78 (2018) no.10, 829.

[37] S. Nojiri and S. D. Odintsov, "Covariant Generalized Holographic Dark Energy and Accelerating Universe," Eur. Phys. J. C 77 (2017) no.8, 528. 
[38] V. Srivastava and U. K. Sharma. "Tsallis holographic dark energy with hybrid expansion law," Int. J. Geom. Methods Mod. Phys. (2020) 2050144, doi:10.1142/S02198878205014443.

[39] A. S. Jahromi, S. A. Moosavi, H. Moradpour, J. P. Morais Graça, I. P. Lobo, I. G. Salako and A. Jawad, "Generalized entropy formalism and a new holographic dark energy model", Phys. Lett. B 780 (2018), 21.

[40] B. Pourhassan, A. Bonilla, M. Faizal and E. M. C. Abreu, "Holographic Dark Energy from Fluid/Gravity Duality Constraint by Cosmological Observations," Phys. Dark Univ. 20 (2018), 41-48.

[41] E. N. Saridakis, "Restoring holographic dark energy in brane cosmology," Phys. Lett. B 660 (2008), 138-143.

[42] G. Varshney, U. K. Sharma, and A. Pradhan, "Reconstructing the $k$-essence and the dilation field models of the THDE in $f(R, T)$ gravity". Eur. Phys. J. Plus 135 (2020), 541 .

[43] S. Nojiri, S. D. Odintsov and E. N. Saridakis, "Holographic inflation," Phys. Lett. B 797 (2019), 134829.

[44] M. Bouhmadi-Lopez, A. Errahmani and T. Ouali, "The cosmology of an holographic induced gravity model with curvature effects," Phys. Rev. D 84 (2011), 083508.

[45] U. K. Sharma, "Reconstruction of quintessence field for the THDE with swampland correspondence in $f(R, T)$ gravity," arXiv:2005.03979 [physics.gen-ph].

[46] Y. Gong and T. Li, "A Modified Holographic Dark Energy Model with Infrared Infinite Extra Dimension(s)," Phys. Lett. B 683 (2010), 241-247.

[47] M. Jamil, E. N. Saridakis and M. R. Setare, "Holographic dark energy with varying gravitational constant," Phys. Lett. B 679 (2009), 172-176.

[48] R. G. Cai, "A Dark Energy Model Characterized by the Age of the Universe," Phys. Lett. B 657 (2007), 228-231.

[49] M. R. Setare and E. C. Vagenas, "Thermodynamical Interpretation of the Interacting Holographic Dark Energy Model in a non-flat Universe," Phys. Lett. B 666 (2008), 111115 .

[50] E. N. Saridakis, "Ricci-Gauss-Bonnet holographic dark energy," Phys. Rev. D 97 (2018) no.6, 064035.

[51] E. N. Saridakis, "Holographic Dark Energy in Braneworld Models with Moving Branes and the w=-1 Crossing," JCAP 04 (2008), 020.

[52] U. K. Sharma and V. C. Dubey, "Exploring the Sharma-Mittal HDE models with different diagnostic tools," Eur. Phys. J. Plus 135 (2020), 391.

[53] R. C. G. Landim, "Holographic dark energy from minimal supergravity," Int. J. Mod. Phys. D 25 (2016) no.04, 1650050. 
[54] C. Q. Geng, Y. T. Hsu, J. R. Lu and L. Yin, "Modified Cosmology Models from Thermodynamical Approach," Eur. Phys. J. C 80 (2020) no.1, 21.

[55] E. N. Saridakis, K. Bamba, R. Myrzakulov and F. K. Anagnostopoulos, "Holographic dark energy through Tsallis entropy," JCAP 12 (2018), 012.

[56] S. M. R. Micheletti, "Observational constraints on holographic tachyonic dark energy in interaction with dark matter," JCAP 05 (2010), 009.

[57] M. Li, X. D. Li, S. Wang and X. Zhang, "Holographic dark energy models: A comparison from the latest observational data," JCAP 06 (2009), 036 [0904.0928 [astro-ph.CO]].

[58] X. Zhang, "Holographic Ricci dark energy: Current observational constraints, quintom feature, and the reconstruction of scalar-field dark energy," Phys. Rev. D 79 (2009), 103509 [0901.2262 [astro-ph.CO]].

[59] R. D'Agostino, "Holographic dark energy from nonadditive entropy: cosmological perturbations and observational constraints," Phys. Rev. D 99 (2019) no.10 103524[1903.03836 [gr-qc]]].

[60] X. Zhang and F. Q. Wu, "Constraints on holographic dark energy from Type Ia supernova observations," Phys. Rev. D 72 (2005), 043524 [astro-ph/0506310].

[61] C. Feng, B. Wang, Y. Gong and R. K. Su, "Testing the viability of the interacting holographic dark energy model by using combined observational constraints," JCAP 09 (2007), 005 [0706.4033 [astro-ph]].

[62] E. Sadri, "Observational constraints on interacting Tsallis holographic dark energy model," Eur. Phys. J. C 79 (2019) no.9, 762 [1905.11210[astro-ph.CO]].

[63] Z. Molavi and A. Khodam-Mohammadi, "Observational tests of Gauss-Bonnet like dark energy model," Eur. Phys. J. Plus 134 (2019) no.6, 254 [1906.05668 [gr-qc]].

[64] J. Lu, E. N. Saridakis, M. R. Setare and L. Xu, "Observational constraints on holographic dark energy with varying gravitational constant," JCAP 03 (2010), 031 [0912.0923 [astroph.CO]].

[65] J. D. Barrow, "The Area of a Rough Black Hole," arXiv:2004.09444 [gr-qc]].

[66] S. Carlip, "Logarithmic corrections to black hole entropy from the Cardy formula," Class. Quant. Grav. 17 (2000), 4175-4186 gr-qc/0005017.

[67] R. K. Kaul and P. Majumdar, "Logarithmic correction to the Bekenstein-Hawking entropy," Phys. Rev. Lett. 84 (2000), 5255-5257

[68] G. Wilk and Z. Wlodarczyk, "On the interpretation of nonextensive parameter q in Tsallis statistics and Levy distributions," Phys. Rev. Lett. 84 (2000), 2770

[69] C. Tsallis and L. J. L. Cirto, "Black hole thermodynamical entropy," Eur. Phys. J. C 73 (2013), 2487 
[70] C. Tsallis, "Possible Generalization of Boltzmann-Gibbs Statistics," J. Statist. Phys. 52 (1988), 479-487

[71] E. N. Saridakis, "Barrow holographic dark energy," arXiv:2005.04115 [gr-qc]].

[72] E. N. Saridakis, "Modified cosmology through spacetime thermodynamics and Barrow horizon entropy," arXiv:2006.01105 [gr-qc]].

[73] E. N. Saridakis and S. Basilakos, "The generalized second law of thermodynamics with Barrow entropy," arXiv:2005.08258 [gr-qc]].

[74] A. A. Mamon, A. Paliathanasis and S. Saha, "Dynamics of an Interacting Barrow Holographic Dark Energy Model and its Thermodynamic Implications," arXiv:2007.16020 $[\mathrm{gr}-\mathrm{qc}]]$.

[75] F. K. Anagnostopoulos, S. Basilakos and E. N. Saridakis, "Observational constraints on Barrow holographic dark energy," Eur. Phys. J. C 80 (2020) 826.

[76] E. M. C. Abreu and J. A. Neto, "Barrow black hole corrected-entropy model and Tsallis nonextensivity," arXiv:2009.10133 [gr-qc]].

[77] E. M. C. Abreu and J. Ananias Neto, "Barrow fractal entropy and the black hole quasinormal modes," Phys. Lett. B 807 (2020) 135602.

[78] E. M. C. Abreu and J. A. Neto, "Thermal features of Barrow corrected-entropy black hole formulation," Eur. Phys. J. C 80 (2020) 776.

[79] S. Vagnozzi, L. Visinelli, O. Mena and D. F. Mota, "Do we have any hope of detecting scattering between dark energy and baryons through cosmology?," Mon. Not. Roy. Astron. Soc. 493, (2020) 1139.

[80] E. Calabrese, R. Putter, D. Huterer, E. V. Linder and A. Melchiorri, "Future CMB constraints on early, cold, or stressed dark energy," Phys. Rev. D 83 (2011) 023011.

[81] P. J. E. Peebles and B. Ratra, "The cosmological constant and dark energy," Rev. Mod. Phys. 75 (2003) 559.

[82] S. Capozziello, "Curvature quintessence," Int. J. Mod. Phys. D11 (2002) 483.

[83] E. Piedipalumbo, M. De Laurentis and S. Capozziello, "Noether symmetries in Interacting Quintessence Cosmology," Phys. Dark Univ. 27 (2020) 100444.

[84] B. A. Bassett, P. S. Corasaniti and M. Kunz, "The Essence of quintessence and the cost of compression," Astrophys. J. Lett. 617 (2004) L1.

[85] I. Zlatev, L. M. Wang and P. J. Steinhardt, "Quintessence, cosmic coincidence, and the cosmological constant," Phys. Rev. Lett. 82 (1999) 896. astro-ph/9807002.

[86] R. R. Caldwell and E. V. Linder, "The limits of quintessence," Phys. Rev. Lett. 95 (2005) 141301; astro-ph/0505494.

[87] X. Zhang, "Reconstructing holographic quintessence," Phys. Lett. B 648 (2007), 1. 